VOL. I (1969), 213-230

\title{
Zero-free fortifying homomorphisms and semigroups of relations
}

\author{
Kenneth D. Magill, Jr
}

\begin{abstract}
In this paper, the concept of a fitted semigroup of binary relations is introduced. The binary operation for these semigroups is ordinary composition of relations. A particular type of homomorphism is investigated which maps a certain kind of subsemigroup of one fitted semigroup into another. The main result states that these homomorphisms are injective and gives a representation for them. This and several related results are then applied to the semigroup of all binary relations on a topological space which have a certain prescribed type of topological property. Compactness is one of these properties and the semigroup $S_{K}[X]$ of all compact binary relations on a Hausdorff $k$-space $X$ is given special attention. It is shown that $X$ and $Y$ are homeomorphic if and only if a number of statements are true which relate the semigroups $S_{K}[X]$ and $S_{K}[Y]$. For example, in order that $X$ and $Y$ be homeomorphic it is both necessary and sufficient that some nonzero ideal of $S_{K}[X]$ be isomorphic to some nonzero ideal of $S_{K}[Y]$.
\end{abstract}

\section{Introduction}

Throughout this paper, ideal means two-sided ideal and we regard any semigroup as an ideal of itself. The kernel of a semigroup is defined to be the intersection of all its ideals if this intersection is not empty $[1, \mathrm{p} .67]$. The semigroups that we investigate in this paper have zero

Received 21 March 1969. Received by J. Austral. Math. Soc. 20 November 1968. Communicated by G.B. Preston 
elements, so in these cases we cannot expect the kernel to give us much information about the semigroup since it is simply the set consisting of the zero element. The smallest nonzero ideal, however, does have some structure and plays an important role in our investigations here.

Consequently, we find it worthwhile to extend the notion of a kernel in the following manner:

DEFINITION 1.1. The base of a semigroup $T$ is defined to be the intersection of all ideals of $T$ if $T$ does not have a zero. In the event $T$ does have a zero and more than one element, the base of $T$ is defined to be the intersection of all ideals which properly contain the zero. The base of the one-element semigroup is defined to be the semigroup itself.

If $T$ has a zero, it can happen that the base consists of the zero and if $T$ has no zero, it is quite possible that the base may be empty. If $T$ has no zero and the base is not empty, then the base coincides with the kernel.

DEFINITION 1.2. A subsemigroup of a semigroup $T$ is referred to as a supported subsemigroup if it contains the base of $T$.

DEFINITION 1.3. A homomorphism from a semigroup $S$ into a semigroup $T$ is a fortifying homomorphism if the image of $S$ is a supported subsemigroup of $T$.

DEFINITION 1.4. A homomorphism from a semigroup $S$ into a semigroup $T$ is a zero-free homomorphism if nonzero elements of $S$ are mapped into nonzero elements of $T$.

We note that any monomorphism from any semigroup $S$ into any semigroup $T$ is zero-free and if $T$ does not happen to contain a zero, then every homomorphism from $S$ into $T$ is zero-free.

In this paper, we continue the study which was begun in [7] by introducing the concept of a fitted semigroup of binary relations. We investigate the zero-free fortifying homomorphisms which map a supported subsemigroup of one fitted semigroup into another. Theorem 2.3 is the main result. It states that such homomorphisms are injective and gives a representation for them. This result and several others which follow from it are applied to the semigroup of all binary relations on a topological space which have a certain prescribed type of topological property. 
Compactness is such a property and the semigroup, under composition, of all compact binary relations on a topological space is studied in more detail. We exhibit a rather extensive class of spaces with the property that any two spaces of the class are homeomorphic if and only if the corresponding semigroups generated by the compact idempotent relations are isomorphic. The analogous problem for semigroups of continuous functions and also semigroups of closed functions was previously treated in [6].

\section{Fitted semigroups and fortifying homomorphisms}

DEFINITION 2.1. A fitted triform is a triple $\left(X, F_{X}, S[X]\right)$ where $X$ is a nonempty set, $F_{X}$ is a nonempty family of subsets of $X, S[X]$ is a family of subsets of $X \times X$, and the following conditions are satisfied: (2.1.1) $H \times\{x\}$ and $\{x\} \times H$ belong to $S[X]$ for each $H \in F_{X}$ and $x \in X$.

(2.1.2) $A \times B \in S[X]$ implies that both $A$ and $B$ belong to $F_{X}$. (2.1.3) $\alpha, \beta \in S[X]$ implies that $\alpha \circ \beta \in S[X]$ where $\alpha \circ \beta$ is defined by

$\alpha \circ \beta=\{(x, y) \in X \times X:(x, z) \in \beta$ and $(z, y) \in \alpha$ for some $z \in X\}$.

One easily verifies that a fitted triform is a triform as defined in [7, Definition (1.1)] and, furthermore, one does not find it difficult to produce examples of triforms which are not fitted. The family of relations $S[X]$ of a fitted triform is a semigroup under composition as defined in (2.1.3) and will be referred to as a fitted semigroup of relations or more often simply as a fitted semigroup. We recall from [7] the following

DEFINITION 2.2. Let $\left(X, F_{X}, S[X]\right)$ and $\left(Y, F_{Y}, S[Y]\right)$ be two triforms. A bijection $h$ from $X$ onto $Y$ is a trimorphism if $h[A] \in F_{Y}$ for each $A \in F_{X}$ and, similarly, $h^{-1}[A] \in F_{X}$ for each $A \in F_{Y}$.

We are now in a position to state the main result of this paper. It gives a representation for a zero-free fortifying homomorphism from a supported subsemigroup of one fitted semigroup into another.

THEOREM 2.3. Let $T$ be a supported subsemigroup of the fitted semigroup $S[X]$ and let $\phi$ be a zero-free fortifying homomorphism from $T$ 
into a fitted semigroup $S[Y]$. Then $\phi$ is injective and there exists a unique trimorphism $h$ from $X$ onto $Y$ such that the following two equivalent conditions are satisfied:

(2.3.1) $\phi(\alpha)=h \circ \alpha \circ h^{-1}$ for each $\alpha \in T$.

(2.3.2) $\phi(\alpha)=\{(h(x), h(y)):(x, y) \in \alpha\}$ for each $\alpha \in T$.

A good portion of the proof of this result will be accomplished through a sequence of lemmas. The first lemma gives information about the base of $S[X]$. Before proving it, we establish some notation and terminology. First of all, it follows from (2.1.1), (2.2.2) and the fact that $F_{X}$ is nonempty that $\{(x, x)\} \in S[X]$ for each $x \in X$. This particular relation plays an important role in the proof of Theorem 2.3 and will be denoted by $(x)$. Secondly, if $X$ has more than one point, then the empty relation must belong to $S[X]$. This relation will be denoted by $\xi$. We note that $\xi$ is the zero of $S[X]$ and that any fortifying homomorphism from a supported subsemigroup of $S[X]$ into $S[Y]$ must take $\xi$ into $\xi$. The domain $D(\alpha)$ and the range $R(\alpha)$ of a relation $\alpha$ are defined by

$$
\begin{aligned}
& D(\alpha)=\{x \in X:(x, y) \in \alpha \text { for some } y \in X\}, \\
& R(\alpha)=\{y \in X:(x, y) \in \alpha \text { for some } x \in X\} .
\end{aligned}
$$

A relation $\alpha$ is said to be rectangular if $\alpha=D(\alpha) \times R(\alpha)$. Now, we determine the base of a fitted semigroup $S[X]$.

LEMMA 2.4. The base of a fitted semigroup $S[X]$ consists of azz elements of $S[X]$ which are rectangular.

Proof. Let $B$ denote the family consisting of all those elements of $S[X]$ which are rectangular. There is only one case where the empty relation does not belong to $S[X]$ and that is when $X=\{x\}, F_{X}=\{\{x\}\}$ and $S[X]=\{(x)\}$. In this particular case, the base of $S[X]$ is $S[X]$ itself which coincides with $B$.

We now consider the remaining case where the empty relation does belong to $S[X]$. Let $\alpha$ be any element of $B-\{\xi\}$. Then $\alpha=A \times B$ for some $A, B \subset X$ and for any $\beta \in S[X]$, one shows that 
$\alpha \circ \beta=C \times B$ where $C=\{x \in D(\beta):(x, y) \in \beta$ for some $y \in A\}$ and

$\beta \circ \alpha=A \times D$ where $D=\{y \in R(\beta):(x, y) \in \beta$ for some $x \in B\}$.

This establishes the fact that $B$ is a two-sided ideal of $S[X]$. Now suppose that $J$ is any two-sided ideal of $S[X]$ which properly contains the zero element $\xi$. Choose any $\alpha \in J-\{\xi\}$ and choose any $(a, b) \in \alpha$, then any nonzero element $B \in B$ is of the form $A \times B$ for some $A, B \subset X$ and we note that

$$
[\{b\} \times B] \circ \alpha \circ[A \times\{a\}]=A \times B=\beta .
$$

It follows from (2.1.2) that both $A$ and $B$ belong to $F_{X}$. Thus, (2.1.1) implies that both $\{b\} \times B$ and $A \times\{a\}$ are elements of $S[X]$ and we conclude that $B \in J$. Therefore, $B \subset J$ and it follows that $B$ is the base of $S[X]$.

LEMMA 2.5. Let $\alpha$ and $\beta$ be two elements of the supported subsemigroup $T$ of $S[X]$. Then $D(\alpha) \subset D(\beta)$ if and only if for each $\gamma \in T, \alpha \circ \gamma \neq \xi$ implies $\beta \circ \gamma \neq \xi$.

Proof. Suppose $D(\alpha) \subset D(\beta)$ and $\alpha \circ \gamma \neq \xi$. Then $(x, y) \in \alpha \circ \gamma$ for some $(x, y)$ and there exists a point $z \in X$ such that $(x, z) \in \gamma$ and $(z, y) \in \alpha$. Thus $z \in D(\alpha) \subset D(\beta)$ and hence, $(z, w) \in \beta$ for some $w \in X$. It follows that $(x, w) \in \beta \circ \gamma$.

On the other hand, if $D(\alpha) \phi D(\beta)$, there exists a point $p \in D(\alpha)-D(\beta)$. By Lemma 2.4, $\langle p\rangle \in T$ and we obtain the desired contradiction by noting that $\alpha \circ\langle p\rangle \neq \xi$ while $\beta^{\circ}\langle p\rangle=\xi$.

In much the same way, one verifies

LEMMA 2.6. $R(\alpha) \subset R(\beta)$ if and only if for each $\gamma \in T, \gamma \circ \alpha \neq \xi$ implies $Y \circ \beta \neq \xi$.

LEMMA 2.7. $D(\alpha) \subset R(B)$ if and only if for each $\gamma \in T-\{\xi\}$, $D(\gamma) \subset D(\alpha)$ implies $\gamma \circ \beta \neq \xi$.

Proof. Suppose $D(\alpha) \subset R(\beta), \gamma \in T-\{\xi\}$ and $D(\gamma) \subset D(\alpha)$. Since $\gamma \neq \xi$, there exists a pair $(x, y) \in \gamma$. Then $x \in D(\gamma) \subset D(\alpha) \subset R(\beta)$ and it follows that $(w, x) \in \beta$ for some $w \in X$. Therefore, $(w, y) \in \gamma \circ \beta$.

If, on the other hand, $D(\alpha) \notin R(\beta)$, then $p \in D(\alpha)-R(\beta)$ for some $p \in X$. Again, Lemma 2.4 implies that $\langle p\rangle \in T-\{\xi\}$ and we see that $D((p)) \subset D(\alpha)$ but $(p) \circ \beta=\xi$. 
Similarly, we have

LEMMA 2.8. $R(\alpha) \subset D(\beta)$ if and only if for each $\gamma \in T-\{\xi\}$, $R(\gamma) \subset R(\alpha)$ implies $B \circ \gamma \neq \xi$.

LEMMA 2.9. $D(\alpha)$ consists of one point if and only if there exists precisely one element $\beta \in T-\{\xi\}$ such that $D(\beta) \subset D(\alpha)$ and $R(\beta) \subset D(\alpha)$.

Proof. It is immediate that if $D(\alpha)$ consists of one point $p$, then $\langle p\rangle$ is the element described in the statement of the lemma.

To establish the sufficiency portion of the proof, suppose that $D(\alpha)$ does not consist of one point. A contradiction is reached immediately by assuming that $\alpha=\xi$. If $\alpha \neq \xi$, then there exist two distinct points $p$ and $q$ in $D(\alpha)$. It follows that $\langle p\rangle,\langle q\rangle \in T-\{\xi\}, D(\langle p\rangle)$, $D(\langle q\rangle) \subset D(\alpha)$ and $R(\langle p\rangle), R(\langle q\rangle) \subset D(\alpha)$. This is a contradiction since $p$ and $q$ are distinct.

The next lemma is easily verified.

LEMMA 2.10. $(x, y) \in \alpha$ if and only if $\langle y\rangle{ }^{\circ} \alpha^{\circ}\langle x\rangle \neq \xi$.

Now let $\phi$ denote the zero-free fortifying homomorphism in the statement of Theorem 2.3. We eventually want to verify that for each $\left.x \in \in^{\prime} X, \phi x\right\rangle=\langle y\rangle$ for some $y \in Y$. As a first approximation, we prove

LEMMA 2.11. For any $x \in X$, we have $D(\phi(x))=R(\phi(x))$.

Proof. Since $\phi[T]$ is a supported subsemigroup of $S[Y]$, we are able to use Lemma 2.7 to show that $D(\phi|x\rangle) \subset R(\phi(x))$. Suppose that $\gamma \in \phi[T]-\{\xi\}$ and $D(\gamma) \subset D(\phi(x))$. We must show that $\gamma \circ \phi(x) \neq \xi$. Let $\alpha$ be any element in $T$ such that $\phi(\alpha)=\gamma$ and suppose that

$D(\alpha) \phi D(\langle x\rangle)$. Then according to Lemma 2.5, there exists an element $\beta \in T$ such that $\alpha \circ \beta \neq \xi$ but $\langle x\rangle \circ \beta=\xi$. Since only the zero of $T$ is mapped into the zero of $S[Y]$, it follows that $\gamma \circ \phi(\beta) \neq \xi$ while $\phi\langle x\rangle \circ \phi(\beta)=\xi$. But this is a contradiction since $D(\gamma) \subset D(\phi(x))$. Consequently, we must conclude that $D(\alpha) \subset D(\langle x\rangle)$, i.e., $D(\alpha)=\{x\}$. It follows that $\alpha \circ\langle x\rangle \neq \xi$ and, hence, that $\phi(\alpha) \circ \phi x\rangle=\gamma \circ \phi(x) \neq \xi$. According to Lemma 2.7 , we have shown that $D(\phi(x)) \subset R(\phi(x))$. Using Lemmas 2.6 and 2.8 , one verifies in much the same way that $R(\phi x x\rangle) \subset D(\phi\langle x\rangle)$. 
LEMMA 2.12. $D(\phi x x\rangle)$ consists of one point.

Proof. According to Lemma 2.9, it is sufficient to show that there exists precisely one element $\beta \in \phi[T]-\{\xi\}$ such that $D(\beta) \subset D(\phi(x))$ and $R(\beta) \subset D(\phi|x\rangle)$. Lemma 2.11 guarantees us the existence of one element which satisfies these conditions, namely $\phi\langle x\rangle$ itself. The proof will be complete when we show that if $\beta$ satisfies these conditions, then $\beta=\phi|x\rangle$. Let $\alpha$ be any element in $T$ such that $\phi(\alpha)=\beta$. We want to show that $\alpha=\langle x\rangle$. Suppose that $D(\alpha) \phi D(\langle x))$. Then by Lemma 2.5, there exists an element $\gamma \in T$ such that $\alpha \circ \gamma \neq \xi$ while $\langle x\rangle \circ \gamma=\xi$. Again we recall that $\phi$ maps only the zero of $T$ into the zero of $S[Y]$ and we conclude that $\beta \circ \phi(\gamma) \neq \xi$ but $\phi(x) \circ \phi(\gamma)=\xi$. This contradicts the fact that $D(\beta) \subset D(\phi x\rangle)$. Thus, we conclude that $D(\alpha) \subset D(\langle x\rangle)$. Using Lema 2.6 and similar techniques, one also shows that $R(\alpha) \subset R(\langle x\rangle)$. Therefore, $\alpha=\langle x\rangle$ and $\beta=\phi(\alpha)=\phi\langle x\rangle$.

LEMMA 2.13. For each $x \in X$, there exists a unique $y \in Y$ such that $\phi\langle x\rangle=\langle y\rangle$.

Proof. Uniqueness is immediate and existence is a consequence of Lemmas 2.11 and 2.12 .

LEMMA 2.14. For each $y \in Y$, there exists an $x \in X$ such that $\phi\langle x\rangle=\langle y\rangle$.

Proof. Since $\phi$ is a fortifying homomorphism, $\phi[T]$ contains the base of $S[Y]$. Consequently, $\phi(\alpha)=\langle y\rangle$ for some $\alpha \in T$. Furthermore, $\alpha \neq \xi$ since $\phi(\alpha) \neq \xi$. Choose any $(a, b) \in \alpha$. Then by Lerma 2.13, there are elements $u, v \in Y$ such that $\phi(a)=\langle u\rangle$ and $\phi b\rangle=\langle v\rangle$. Since $\langle b\rangle \circ \alpha \circ(a) \neq \xi$, it follows that

$$
\langle v\rangle \circ\langle y\rangle \circ\langle u\rangle=\phi(b\rangle \circ \phi(\alpha) \circ \phi(a) \neq \xi \text {. }
$$

But this implies that $v=y=u$ and it follows that $\phi\langle a\rangle=\langle y\rangle$.

Lemmas $2.10,2.13$ and 2.14 give us the facts we need in order to complete the proof of Theorem 2.3. According to Lemma 2.13, there exists, for each $x \in X$, a unique $y \in Y$ such that $\phi(x)=\langle y\rangle$. We define a mapping $h$ from. $X$ into $Y$ by $h(x)=y$ and we note that

$$
\phi x\rangle=\langle h(x)\rangle
$$

for each $x \in X$. On the other hand, Lemma 2.14 assures us that there 
exists, for each $y \in Y$, at least one $x \in X$ such that $\phi\langle x\rangle=\langle y\rangle$. Choose any such $x$ with this property and define a mapping $k$ from $Y$ into $X$ by $k(y)=x$. We note that

$$
\phi\langle k(y)\rangle=\langle y\rangle
$$

for each $y \in Y$. Now we show that

$$
\phi(\alpha)=h \circ \alpha \circ k
$$

for each $\alpha \in T$.

First, suppose that $(u, v) \in \phi(\alpha)$. By Lemma 2.14, there exists at least one $x \in X$ such that $\phi\langle x\rangle=\langle v\rangle$. Then because of Lemma 2.10 and $(2.14 .2)$, we have

$$
\xi \neq\langle v\rangle \circ \phi(\alpha) \circ\langle u\rangle=\phi\langle x\rangle \circ \phi(\alpha) \circ \phi\langle k(u)\rangle=\phi(\langle x\rangle \circ \alpha \circ\langle k(u)\rangle) .
$$

This implies that $\langle x\rangle \circ \alpha \circ\langle k(u)\rangle \neq \xi$ and using Lemma 2.10 once again, it follows that $(k(u), x) \in \alpha$. Since $\phi\langle x\rangle=\langle v\rangle$, it follows from (2.14.1) that $h(x)=v$. All this implies that $(u, v) \in h \circ \alpha \circ k$.

On the other hand, suppose that $(u, v) \in h \circ \alpha \circ k$. Then there exist elements $w, x \in X$ such that $(u, w) \in k,(w, x) \in \alpha$ and $(x, v) \in h$. Since $k(u)=w$ and $h(x)=v$, it follows from (2.14.1) and $(2.14 .2)$ that $\phi(x)=\langle v\rangle$ and $\phi\langle w\rangle=\langle u\rangle$. Since $(w, x) \in \alpha$, Lemma 2.10 implies that $\langle x\rangle \circ \alpha \circ\langle w\rangle \neq \xi$ and since $\phi$ maps only the zero of $T$ into the zero of $S[Y]$, we have

$$
\xi \neq \phi(\langle x\rangle \circ \alpha \circ\langle w\rangle)=\phi\langle x\rangle \circ \phi(\alpha) \circ \phi\langle w\rangle=\langle v\rangle \circ \phi(\alpha) \circ\langle u\rangle .
$$

By appealing once again to Lemma 2.10, we are able to conclude that $(u, v) \in \phi(\alpha)$.

$$
\begin{gathered}
\text { Using both (2.14.1) and (2.14.2) we see that for any } y \in Y, \\
\qquad h(k(y))\rangle=\phi\langle k(y)\rangle=\langle y\rangle .
\end{gathered}
$$

This implies that $h(k(y))=y$, i.e., $h \circ k$ is the identity map on $Y$. It also happens that $k \circ h$ is the identity map on $X$. We verify this by assuming otherwise and deriving a contradiction. Suppose there exists a point $p \in X$ such that $k(h(p))$. We then choose any $y \in Y$ and note that $(2.14 .4) \phi\langle p\rangle \circ \phi\{(k(y), p)\}=h \circ\langle p\rangle \circ k \circ h \circ\{(k(y), p)\} \circ k=\xi$ and 
$(2.14 .5) \quad \phi(\langle p\rangle \circ\{(k(y), p)\})=h \circ(p\rangle \circ\{(k(y), p)\} \circ k \neq \xi \circ$

The former follows from the fact that $k(h(p)) \notin D(\langle p\rangle)$ and the latter is a consequence of the fact that

$$
(y, h(p)) \in h \circ\langle p\rangle \circ\{(k(y), p)\} \circ k \text {. }
$$

Statements $(2.14 .4)$ and $(2.14 .5)$ together imply that

$$
\phi\langle p\rangle \circ \phi\{(k(y), p)\} \neq \phi(\langle p\rangle \cdot\{(k(y), p)\})
$$

which is the contradiction we seek. Thus, $k \circ h$ must be the identity map on $X$. It follows that $h$ is bijective and $k=h^{-1}$. Therefore, we may rewrite $(2.14 .3)$ as

$$
\phi(\alpha)=h \circ \alpha \circ h^{-1}
$$

for each $\alpha \in T$. It follows immediately from this that $\phi$ is injective.

Now let $A$ be any subset of $X$ which belongs to $F_{X}$. Then for any $p \in X, A \times\{p\}$ belongs to $S[X]$ by (2.1.1) and it follows from (2.14.6) that

$$
\phi(A \times\{p\})=h \circ(A \times\{p\}) \circ h^{-1}=h[A] \times\{h(p)\} .
$$

Hence, $h[A] \times\{h(p)\}$ belongs to $S[Y]$ and it follows from (2.1.2) that $h[A] \in F_{Y}$.

On the other hand, suppose $A \in F_{Y}$. Then for any $q \in Y$, $A \times\{q\} \in S[Y]$ and since $\phi$ is a fortifying homomorphism, Lemma 2.4 implies that $A \times\{q\} \in \phi[T]$. Then

$$
\phi^{-1}(A \times\{q\})=h^{-1} \circ(A \times\{q\}) \circ h=h^{-1}[A] \times\left\{h^{-1}(q)\right\}
$$

belongs to $T$ and it then follows from (2.1.2) that $h^{-1}[A] \in F_{X}$. This proves that the mapping $h$ is a trimorphism.

To prove that $h$ is unique, suppose that $t$ is any bijection from $X$ onto $y$ with the property that $\phi(\alpha)=t \circ \alpha \circ t^{-1}$ for each $\alpha \in T$. Then, in particular,

$$
\phi\langle x\rangle=t \circ\langle x\rangle \circ t^{-1}=\langle t(x)\rangle
$$

for each $x \in X$. Since we also have 


$$
\phi\langle x\rangle=h \circ\langle x\rangle \circ h^{-1}=\langle h(x)\rangle,
$$

it follows that $t(x)=h(x)$ for each $x \in X$. Thus $t$ and $h$ are identical. The only thing which remains is to show that

$$
h \circ \alpha \circ h^{-1}=\{(h(x), h(y)):(x, y) \in \alpha\} \text {. }
$$

The verification is straightforward and will be omitted.

In the proof of Theorem 2.3, the fact that the homomorphism was zero-free played a very essential role. The following example shows that we cannot dispense with this condition and hope to prove the resulting statement.

EXAMPLE 2.15. Let $X$ denote the semigroup, under composition, of all binary relations on a nonempty set $Y$ and let $F(X)$ denote the semigroup, under composition, of all functions which map $X$ into $X$. We define a mapping $\psi$ from $X$ into $F(X)$ by

$$
(\psi(\alpha))(\beta)=\alpha \circ \beta,
$$

that is, for each $\alpha \in X, \psi(\alpha)$ is the function in $E(X)$ which maps $\beta$ into $\alpha \circ \beta$. It is easily verified that the mapping $\psi$ is a monomorphism. Now let

$$
T=\psi[X] \cup A
$$

where $A$ denotes the base of $B(X)$, the semigroup of all binary relations on $X$. We define a mapping $\phi$ from $T$ into $X$ by

$$
\begin{array}{lll}
\phi(\alpha)=\psi^{-1}(\alpha) & \text { for each } & \alpha \in \psi[X], \\
\phi(\alpha)=\xi & \text { for each } & \alpha \in A .
\end{array}
$$

One easily checks that $\phi$ is a homomorphism from $T$ onto $X$. Furthermore, $T$ is a supported subsemigroup of $B(X)$ and since the image of $T$, under $\phi$, is all of $X$, the mapping $\phi$ is a fortifying homomorphism which is not zero-free. Since $\phi$ is not injective, it cannot have a representation as in (2.3.1) or (2.3.2).

The next result is a consequence of Theorem 2.3 and the fact that any semigroup is a supported subsemigroup of itself.

COROLLARY 2.16. Let $S[X]$ and $S[Y]$ be two fitted semigroups and let $\phi$ be a zero-free homomorphism from $S[X]$ onto $S[Y]$. Then $\phi$ is an 
isomorphism and there exists a unique trimorphism $h$ from $X$ onto $Y$ such that the following two equivalent conditions are satisfied:

$$
\begin{array}{lll}
(2.16 .1) & \phi(\alpha)=h \circ \alpha \circ h^{-1} & \text { for each } \alpha \in S[X], \\
(2.16 .2) & \phi(\alpha)=\{(h(x), h(y)):(x, y) \in \alpha\} & \text { for each } \alpha \in S[X] .
\end{array}
$$

COROLLARY 2.17. Let $S[X]$ be a fitted semigroup. Then the automorphism group of any supported subsemigroup of $S[X]$ is isomorphic to a subgroup of the group, under composition, of all trimorphisms which map $x$ onto $X$.

Proof. Let $T$ be a supported subsemigroup of $S[X]$ and let $\phi$ be an automorphism of $T$. According to Theorem 2.3, there exists a unique trimorphism $h$ mapping $X$ onto $X$ such that $\phi(\alpha)=h \circ \alpha^{\circ} h^{-1}$ for each $\alpha \in T$. One can show that the mapping $\Phi$ defined by $\Phi(\phi)=h$ is a monomorphism from the automorphism group of $T$ into the group of all trimorphisms on $X$.

DEFINITION 2.18. The symbol $I[X]$ is used to denote the subsemigroup of the fitted semigroup $S[X]$ which is generated by the idempotent elements of $S[X]$.

LEMMA 2.19. Let $\left(X, F_{X}, S[X]\right)$ be a fitted triform with the additional property that all two-element subsets of $X$ belong to $F_{X}$. Then $I[X]$ is a supported subsemigroup of $S[X]$.

Proof. It is immediate that if $\xi \in S[X]$, then $\xi \in I[X]$, so let $\alpha$ be any element of the base of $S[X]$ which is different from $\xi$. By Lemma 2.4, $\alpha=A \times B$ for two nonempty subsets $A$ and $B$ of $X$. Choose $a \in A$ and $b \in B$. Since $A \times B \in S[X]$, condition (2.1.2) implies that both $A$ and $B$ belong to $F_{X}$. It then follows from condition (2.1.1) that $\beta=\{b\} \times B$ and $\rho=A \times\{a\}$ both belong to $S[X]$. Furthermore, $\{a, b\}$ belongs to $F_{X}$ by hypothesis and another appeal to (2.1.1) allows us to conclude that

$$
\gamma=\{a, b\} \times\{b\}=\{(a, b),(b, b)\}
$$

belongs to $S[X]$. One easily verifies that $\beta, \gamma$ and $\rho$ are all idempotent and it follows that $\alpha \in I[X]$ since $\alpha=\beta \circ \gamma \circ \rho$. 
Now suppose that $\left(X, F_{X}, S[X]\right)$ and $\left(Y, F_{Y}, S[Y]\right)$ are fitted triforms such that $F_{X}$ contains all two-element subsets of $X$ and $F_{Y}$ contains all two-element subsets of $Y$. Then by Lemma 2.19, any homomorphism from $I[X]$ onto $I[Y]$ may be regarded as a fortifying homomorphism from a supported subsemigroup of $S[X]$ into $S[Y]$. These observations, together with Theorem 2.3 yield

THEOREM 2.20. Let $\left(X, F_{X}, S[X]\right)$ and $\left(Y, F_{Y}, S[Y]\right)$ be two fitted triforms such that $F_{X}$ contains all two-element subsets of $X$ and $F_{Y}$ contains all two-elements of $Y$. Furthermore, let $\phi$ be a zero-free homomorphism from $I[X]$ onto $I[Y]$. Then $\phi$ is, in fact, an isomorphism and there exists a unique trimorphism $h$ from $X$ onto $Y$ such that each of the following two equivalent conditions are satisfied:

$$
\begin{array}{lll}
(2.20 .1) & \phi(\alpha)=h \circ \alpha \circ h^{-1} & \text { for each } \alpha \in I[X], \\
(2.20 .2) & \phi(\alpha)=\{(h(x), h(y)):(x, y) \in \alpha\} & \text { for each } \alpha \in I[X] .
\end{array}
$$

\section{Semigroups of relations on topological spaces}

Here, we apply the results of the previous section to various semigroups of relations on topological spaces. We make the blanket assumption that all topological spaces discussed in this paper are Hausdorff. Let us agree (as in [8]) to regard a topological property $Q$ as a class of spaces such that if $X \in Q$ and $Y$ is homeomorphic to $X$, then $Y \in Q$. Let us agree further that the statement " $X$ has property $Q$ " is equivalent to the statement $" X \in Q$ ".

DEFINITION 3.1. A bijection $h$ from a topological space $X$ onto a topological space $Y$ is a $Q$-morphism if $h[A] \subset Y$ has property $Q$ whenever $A \subset X$ has property $Q$ and similarly, $h^{-1}[A] \subset X$ has property $Q$ whenever $A \subset Y$ has property $Q$.

DEFINITION 3.2. A topological property $Q$ is said to be composable if all points have property $Q$ and for any space $X$, if $\alpha, \beta \subset X \times X$ both have property $Q$, then $\alpha \circ \beta$ has property $Q$.

DEFINITION 3.3. A topological property $Q$ is said to be factorable if for any two spaces $X$ and $Y$, both $X$ and $Y$ have property $Q$ 
whenever $X \times Y$ has property $Q$.

The notion of a composable topological property was introduced in [8]. Among other things, it was shown there that compactness and $\sigma$-compactness are composable topological properties while normality and connectedness are not.

Suppose $Q$ is a topological property which is both factorable and composable and let $X$ be any topological space. In keeping with the notation in [8], we let $Q_{X}$ denote the family of all subsets of $X$ which have property $Q$ and we let $S_{Q}[X]$ denote the semigroup, under composition, of all binary relations on $X$ which have property $Q$. For any subset $H$ of $X$ and any point $x \in X$, both $H \times\{x\}$ and $\{x\} \times H$ are homeomorphic to $H$ and consequently, both belong to $S_{Q}[X]$ if $H$ belongs to $Q_{X}$. Furthermore, since $Q$ is factorable, both $A$ and $B$ must belong to $Q_{X}$ if $A \times B \in S_{Q}[X]$. Therefore, the triple $\left(X, Q_{X}, S_{Q}[X]\right)$ is a fitted triform whenever the property $Q$ is both factorable and composable. An application of Theorem 2.3 now yields

THEOREM 3.4. Let $Q$ be a topological property which is both factorable and composable and let $S_{Q}[X]$ and $S_{Q}[Y]$ denote the semigroups, under composition, of all those binary relations on the spaces $X$ and $Y$ respectively which have property $Q$. Let $\phi$ be a zero-free fortifying homomorphism from a supported subsemigroup $T$ of $S_{Q}[X]$ into $S_{Q}[Y]$. Then $\phi$ is injective and there exists a unique Q-morphism $h$ mapping $X$ onto $Y$ such that the following two equivalent conditions are satisfied:

(3.4.1) $\phi(\alpha)=h \circ \alpha \circ h^{-1}$ for each $\alpha \in T$, $\phi(\alpha)=\{(h(x), h(y)):(x, y) \in \alpha\}$ for each $\alpha \in T$.

COROLLARY 3.5. Let $Q$ be a topological property which is both factorable and composable, let $X$ and $Y$ be any two topological spaces, and let $\phi$ be a zero-free homomorphism from $S_{Q}[X]$ onto $S_{Q}[Y]$. Then $\phi$ is an isomorphism and there exists a unique Q-morphism $h$ mapping $X$ onto $Y$ such that the following two equivalent conditions are satisfied: 


$$
\begin{array}{lll}
(3.5 .1) & \phi(\alpha)=h \circ \alpha \circ h^{-1} & \text { for each } \alpha \in S_{Q}[X], \\
(3.5 .2) & \phi(\alpha)=\{(h(x), h(y)):(x, y) \in \alpha\} & \text { for each } \alpha \in S_{Q}[X] .
\end{array}
$$

An application of Corollary 2.17 immediately results in

COROLLARY 3.6. Let $Q$ be a topological property which is both factorable and composable and let $X$ be any topological space. Then the automorphism group of any supported subsemigroup of $S_{Q}[X]$ is isomorphic to a subgroup of the group of all Q-morphisms on $X$.

DEFINITION 3.7. For any composable topological property $Q$ and any topological space $X, I_{Q}[X]$ denotes the semigroup, under composition, which is generated by the idempotent relations on $X$ which have property $Q$.

With respect to the semigroup $I_{Q}[X]$, Theorem 2.20 translates into

THEOREM 3.8. Let $Q$ be a topological property which is both factorable and composable and, furthermore, is such that the two-element space has property $Q$. Then for any two spaces $X$ and $Y$, any zero-free homomorphism $\phi$ from $I_{Q}[X]$ onto $I_{Q}[Y]$ is, in fact, an isomorphism and there exists a unique Q-morphism $h$ from $X$ onto $Y$ such that the following two equivalent conditions are satisfied:

$$
\begin{array}{lll}
(3.8 .1) & \phi(\alpha)=h \circ \alpha \circ h^{-1} & \text { for each } \alpha \in I_{Q}[X], \\
(3.8 .2) & \phi(\alpha)=\{(h(x), h(y)):(x, y) \in \alpha\} & \text { for each } \alpha \in I_{Q}[X] .
\end{array}
$$

Now we consider the special case where the topological property is compactness. As we mentioned previously, compactness is composable [8, Theorem (2.6)]. Since, in addition, compactness is factorable and the two-element space is compact, all the results obtained thus far in this section can be applied. We denote the compactness property by $K$. For any space $X$, we let $S_{K}[X]$ denote the semigroup, under composition, of all compact binary relations on $X$ and we let $I_{K}[X]$ denote the semigroup which is generated by the idempotent compact binary relations on $X$. We are able to get sharper results without sacrificing a great deal if we restrict ourselves to $k$-spaces [2, p. 230-231]. A space is defined to be a $k$-space if it has the property that any subset which intersects each compact subset 
in a closed subset must, itself, be closed. The class of all $k$-spaces is fairly extensive in that it includes all locally compact spaces as well as all first countable spaces [2, p. 231, Theorem 13]. An important fact about the class of $k$-spaces is that any $K$-morphism between two such spaces must actually be a homeomorphism. Thus, in Theorems 3.4 and 3.8 , the $Q$-morphism $h$ is a homeomorphism if $Q$ happens to be compactness and $X$ and $Y$ are $k$-spaces.

THEOREM 3.9. Let $X$ and $Y$ be two nonempty $k$-spaces. Then the following statements about a zero-free homomorphism $\phi$ from $S_{K}[X]$ into $S_{K}[Y]$ are equivalent:

(3.9.1) The image of $S_{K}[X]$ contains a nonzero ideal of $S_{K}[Y]$.

(3.9.2) $\phi$ is a fortifying homomorphism.

(3.9.3) $\phi$ is a homomorphism from $S_{K}[X]$ onto $S_{K}[Y]$.

(3.9.4) $\phi$ is an isomorphism from $S_{K}[X]$ onto $S_{K}[Y]$.

(3.9.5) There exists a unique homeomorphism $h$ from $X$ onto $Y$ such that $\phi(\alpha)=h \circ \alpha \circ h^{-1}$ for each $\alpha \in S_{K}[X]$ or, equivalently, $\phi(\alpha)=\{(h(x), h(y)):(x, y) \in \alpha\}$ for each $\alpha \in S_{K}[X]$.

Proof. It is immediate that (3.9.4) implies (3.9.3) and (3.9.3) implies (3.9.1). Furthermore, since any subsemigroup of $S_{K}[Y]$ which contains a nonzero ideal of $S_{K}[Y]$ is a supported subsemigroup, we see that (3.9.1) implies (3.9.2). As we mentioned previously, any $K$-morphism between $k$-spaces is a homeomorphism and thus, it follows from Theorem 3.4 that (3.9.2) implies (3.9.5). It remains for us to observe that (3.9.5) implies (3.9.4). But it follows quite easily that any homomorphism from $S_{K}[X]$ into $S_{K}[Y]$ which has a representation as in $(3.9 .5)$ must actually be an isomorphism onto $S_{K}[Y]$.

THEOREM 3.10. For any two nonempty $k$-spaces $X$ and $Y$, the following statements are equivalent: 
(3.10.1) $X$ and $Y$ are homeomorphic.

(3.10.2) $S_{K}[X]$ and $S_{K}[Y]$ are isomorphic.

(3.10.3) $S_{K}[Y]$ is the image of $S_{K}[X]$ under a zero-free homomorphism.

(3.10.4) Some nonzero ideal of $S_{K}[Y]$ is isomorphic to an ideal of $S_{K}[X]$

(3.10.5) Some nonzero ideal of $S_{K}[Y]$ is the image of an ideal of $S_{K}[X]$ under a zero-free homomorphism.

(3.10.6) $I_{K}[X]$ and $I_{K}[Y]$ are isomorphic.

(3.10.7) $I_{K}[Y]$ is the image of $I_{K}[X]$ under a zero-free homomorphism.

Proof. It follows from the previous theorem that (3.10.1), (3.10.2) and $(3.10 .3)$ are all equivalent and it follows from Theorem 3.8 that (3.10.6) and (3.10.7) are equivalent. Furthermore, since any nonzero ideal of $S_{K}[Y]$ is a supported subsemigroup of $S_{K}[Y]$, it follows from Theorem 3.4 that $(3.10 .4)$ and $(3.10 .5)$ are equivalent. Because of all this, the proof will be complete when we show that (3.10.2), (3.10.4) and (3.10.6) are equivalent. It is immediate that (3.10.2) implies both (3.10.4) and (3.10.6) and it follows from Theorem 3.4 and Theorem 3.8 respectively that $(3.10 .4)$ and $(3.10 .6)$ both imply (3.10.1) (we recall again that between $k$-spaces, a $K$-morphism is a homeomorphism). Since, for any homeomorphism $h$ from $X$ onto $Y$, the mapping which takes $\alpha \in S_{K}[X]$ into $h \circ \alpha \circ h^{-1} \in S_{K}[Y]$ is an isomorphism from $S_{K}[X]$ onto $S_{K}[Y]$, it follows that (3.10.1) implies (3.10.2).

\section{Some concluding remarks}

In [6], we treated the problem of finding a nontrivial class $C$ of spaces with the property that any two spaces of the class are homeomorphic if and only if the two corresponding semigroups generated by the idempotent continuous selfmaps are isomorphic. It was shown there that the class consisting of all O-dimensional (Hausdorff) spaces and all normal (Hausdorff) spaces which contain an arc is such a class. It was also shown 
that the class of all $T_{1}$ spaces played the same role for the semigroups generated by the idempotent closed selfmaps. Theorem 3.10 provides a solution to the analogous problem for the semigroups $I_{K}[X]$ which are generated by the idempotent compact relations on $X$ for, among other things, this result implies that any two $k$-spaces are homeomorphic if and only if the two corresponding semigroups which are generated by the idempotent compact relations are isomorphic. As we mentioned previously, the class of $k$-spaces is fairly extensive as it contains all locally compact spaces and all first countable spaces.

Finally, we wish to note that the class $K$ of semigroups of compact relations on $k$-spaces has an interesting property which is not shared by the class $V$ of semigroups of continuous selfmaps on $S^{*}$-spaces [5] or the class $W$ of elosed selfmaps on a $T_{1}$ space [4]. The property is simply this: the algebraic structure of any semigroup in the class $K$ is completely determined within that class by the algebraic structure of any one of its nonzero ideals. That is, if any two of the semigroups in $K$ have isomorphic nonzero ideals, then the semigroups themselves must be isomorphic. This fact follows from Theorem 3.10. However, the base (which in this case coincides with the kernel) of any semigroup in $V$ consists of all the constant functions and is therefore a left zero semigroup. Thus, the bases of any two such semigroups are isomorphic if the two corresponding topological spaces have the same cardinality. But the semigroups themselves will not be isomorphic unless the spaces are homeomorphic [5, p. 295, Theorem 1]. See also [9]. Similar remarks are valid for semigroups from the class $w$.

\section{References}

[1] A.H. Clifford and G.B. Preston, The algebraic theory of semigroups, vols. 1 and 2, (Amer. Math. Soc. Mathematical Surveys 7 , Providence, 1961 and 1967).

[2] John L. Kelley, General topology, (Van Nostrand, Princeton, 1955).

[3] E.S. Ljapin, Semigroups (Translations of Mathematical Monographs, vol. 3, Amer. Math. Soc. 1963).

[4] Kenneth D. Magill, Jr, "Semigroups of functions on topological spaces", Proc. London Math. Soc. (3) 16 (1966), 507-518. 
[5] Kenneth D. Magill, Jr, "Another S-admissible class of spaces", Proc. Amer. Math. Soc. 18 (1967), 295-298.

[6] K.D. Magill, Jr, "Semigroups of functions generated by idempotents", $J$. London Math. Soc. 44 (1969), 236-242.

[7] Kenneth D. Magill, Jr, "Isomorphisms of triform semigroups", J. Austral. Math. Soc. (to appear).

[8] Kenneth D. Magill, Jr, "Composable topological properties and semigroups of relations", J. Austral. Math. Soc. (to appear).

[9] A.A. Mal'cev, "A remark of a theorem of M. Gavrilov", (Russian) Taškent. Politehn. Inst. Naūn. Trudy (Novaja Serija) No. 37 (1966), 30-32.

Department of Mathematics, State University of New York at Buffalo, USA. 